Appl. Phys. A 48, 143-147 (1989)

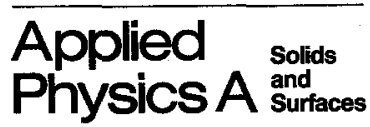

(C) Springer-Verlag 1989

\title{
Sequence of Different Types of Nonlinear Current Oscillation in n-GaAs
}

\author{
J. Spangler, A. Brandl, and W. Prettl \\ Naturwissenschaftliche Fakultät II - Physik, Universität, D-8400 Regensburg, \\ Fed. Rep. Germany
}

Received 28 April 1988/Accepted 22 September 1988

\begin{abstract}
Self-generated chaotic current fluctuations in the post-breakdown regime of a $\mathrm{n}$-GaAs layer at $4.2 \mathrm{~K}$ have been analyzed in detail. Without an external magnetic field only regular oscillations were observed. Increasing the magnetic field strength up to $100 \mathrm{mT}$ generates a sequence of quasiperiodic and frequency-locking current oscillations and finally a Ruelle-Takens-Newhouse scenario with chaos. This may be understood by assuming two coupled oscillatory processes caused by dielectric relaxation and energy relaxation in the distribution of free carriers.
\end{abstract}

PACS: 05.40., 72.70., 72.20J

High-purity semiconductors at low temperature show highly nonlinear current-voltage characteristics. For small electric fields almost all carriers are bound to shallow impurities yielding a low conductance of the sample. At a critical field of a few volts per $\mathrm{cm}$ the impact ionization rate of shallow impurities exceeds the capture rate for low carrier concentration resulting in a rapid increase of the current. The steady-state properties of the transition from the low-conducting state to the high-conducting state have been analyzed in terms of nonequilibrium phase transformations [1-4]. In the course of the transition, spontaneous oscillations and chaotic current fluctuations have been observed in several semiconductor materials [5-17]. Different types of, and routes to, chaos were recognized and discussed in terms of nonlinear dynamics [18-28]. Current fluctuations in semiconductors may occur spontaneously [5-13] or be induced by an external periodic driving force [13-17].

In the present paper we report on a detailed study of self-generated current fluctuations in high purity $\mathrm{n}-\mathrm{GaAs}$ epitaxial layers which occur within a limited bias voltage interval in the post-breakdown regime of the material. The observed phenomena depend critically on the strength of an external magnetic field. At zero field, $B=0$, only regular oscillations were found. Increasing $B$ up to not more than $100 \mathrm{mT}$ causes a sequence of quasiperiodic and frequency-locking cur- rent phenomena, finally undergoing a Ruelle-TakensNewhouse scenario to chaos. This behavior may be attributed to the coupling of two oscillatory processes, in the present case dielectric relaxation and an oscillation of the nonequilibrium electron distribution. The experimental results are in excellent agreement with the predictions of the circle-map theory. The coupling strength, frequencies and amplitudes of both selfsustained processes depend strongly on the magnetic field strength.

\section{Experimental Setup}

The measurements were carried out on a n-GaAs epitaxial layer grown by liquid phase epitaxy on a Crcompensated substrate. Ohmic point contacts were formed $2 \mathrm{~mm}$ apart from each other on the $16 \mu \mathrm{m}$ thick layer. The material characteristics of the sample are: carrier concentration $n=1.3 \times 10^{14} \mathrm{~cm}^{-3}$, electron mobility $\mu=8.9 \times 10^{4} \mathrm{~cm}^{2} / \mathrm{Vs}$ at $77 \mathrm{~K}$ corresponding to a donor concentration $N_{\mathrm{D}}=5.7 \times 10^{14} \mathrm{~cm}^{-3}$ and a compensation ratio of $77 \%$. The sample was immersed in liquid helium and was shielded against ambient and thermal radiation. Using a superconducting solenoid a magnetic field $B$ was applied perpendicular to the sample surface and the current through the layer. The bias voltage was applied with a load resistor of $200 \mathrm{k} \Omega$, and, as a probe of the current, the voltage across the sample was measured. 


\section{Current-Voltage Characteristics}

In order to localize regions in the current-voltage plane where fluctuations of a certain type occur, the currentvoltage characteristic for various magnetic field strengths were recorded. Current-voltage (I-V) characteristics are usually measured by averaging the fluctuations. This yields the well-known nonlinear $\mathrm{I}-\mathrm{V}$ relations showing negative differential resistance, sharp kinks and hysteresis. These structures in the I-V characteristic depend on the amplitude of the fluctuations as well as on the shape of the oscillations as a function of time, which may be strongly affected by the external circuitry. To get rid of these artefacts we present a new method to visualize current-voltage characteristics with oscillatory regions. The sample was biased in series with a sufficiently large load resistor to have constant current conditions, and the voltage fluctuations across the sample were measured by applying broad-band electronics. In the currentvoltage plane the extrema of the voltage oscillations and the average voltage as a function of the current were recorded. The results are displayed in Fig. 1a where hatched regions show the extent of the fluctuation amplitudes.

In these areas of the $\mathrm{I}-\mathrm{V}$ plane virtually no definite relation between current and voltage exists. The oscillatory regimes of the $\mathrm{I}-\mathrm{V}$ plane are different for increasing and decreasing current which is indicated by right and left inclined hatching in Fig. 1a.

The measurements show that in the area of the I-V plane where oscillations occur, two different regimes may be distinguished, that with large voltage amplitudes and that with small amplitudes. Increasing the magnetic field strength the large amplitude region shrinks on the current scale and new types of oscillation with small amplitudes appear for high currents. The large amplitudes decrease slightly and the small amplitudes increase drastically with rising magnetic field strength. For higher magnetic fields (not shown in Fig. 1) the large amplitude region totally vanishes. The results of the present investigation were obtained for currents at the top of and above the large voltage amplitude area inside the small amplitude region.

Averaging the fluctuations yields typical currentvoltage characteristics with the well-known hysteresis effects as shown in Fig. 1 b.

Comparison between the two kinds of recording proves that current-voltage hysteresis is not a steadystate property of the sample; rather, it is due simply to a change in the character of the fluctuations.

\section{Spontaneous Oscillations}

To analyze the spontaneous oscillations, time series of the voltage across the sample were digitally recorded with sampling intervals $\Delta t$ ranging from 50 to $5000 \mathrm{~ns}$. The recorded signals were used to calculate power spectra, phase portraits, Poincaré sections and the fractal dimension $d$ of the fluctuations as functions of the voltage applied to the sample and the load resistor in series. Because of the strong fluctuations in the oscillatory regime, the bias voltage of the sample itself could not be taken as a control parameter. The power spectra were obtained by averaging 20 independent spectra of different time series consisting of 2048 sampling points.

At $B=0$, regular oscillations were found, which set in just above the breakdown voltage and remain
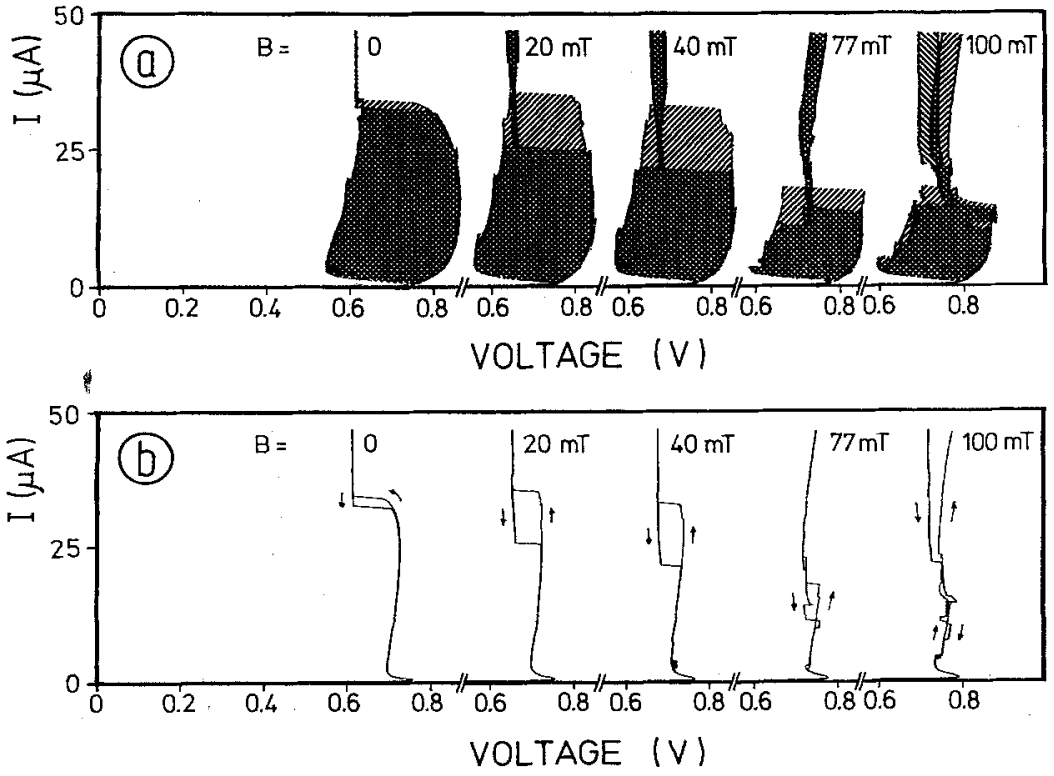

Fig. 1a, b. Current-voltage characteristics for various applied magnetic fields $B$ (load resistor $R_{\mathrm{L}}=1 \mathrm{M} \Omega$ bath temperature $T=4.2 \mathrm{~K}$ ): a New method marking the oscillatory regime (from bottom-left to topright inclined hatching denotes increasing current, from top-left to bottom-right inclined batching denotes decreasing current); b averaged current-voltage characteristics 
coherent up to well above breakdown. Their frequency increases up to $5 \mathrm{MHz}$ with rising voltage.

The observed spectral features of $B>0$ are summarized in Fig. 2 for the voltage range 5 to $7 \mathrm{~V}$ in which all the essential effects occurred.

In all cases a fundamental relaxation oscillation of frequency $f_{1}$ belonging to the small amplitude class was present. Therefore, all frequencies in Fig. 2 are given in units of $f_{1}$. Between $B=16 \mathrm{mT}$ and $40 \mathrm{mT}$ the relaxation oscillations are modified by the occurrence of a second frequency indicating a quasiperiodic behavior of the oscillations. Measurements for $40 \mathrm{mT}$ are displayed in Fig. 2a. The fundamental frequency is practically constant $f_{1}=4.1 \mathrm{MHz}$ for the total voltage range. The $f_{2}$ oscillation sets in at $6.5 \mathrm{~V}$ with $f_{2}=890 \mathrm{kHz}$ being smaller than $f_{1}$ and disappears at $7 \mathrm{~V}$. Poincaré sections revealed a torus type attractor and the analysis of the retun map confirmed the quasiperiodicity of the oscillations.

At $B=77 \mathrm{~m}$ T the onset of the $f_{2}$ oscillation occurs at $5.7 \mathrm{~V}$ and the frequency $f_{2}$ is larger than the $f_{1}$ fundamental frequency (now $f_{1}$ is about $700 \mathrm{kHz}$ ) as shown in Fig. $2 \mathrm{~b}$. Increasing the bias voltage again reduces $f_{2}$, however not continuously as in the case of $B=40 \mathrm{mT}$, but by locking to rational fractions of $f_{1}$. The frequency ratios follow the Farey tree $[29] f_{2} / f_{1}$ $=5 / 2,7 / 3,9 / 4$, and $11 / 5$ with decreasing locking intervals on the voltage scale. The frequency $f_{1}$ decreases slightly from $713 \mathrm{kHz}$ at a voltage of $5.75 \mathrm{~V}$ to $688 \mathrm{kHz}$ at $6.84 \mathrm{~V}$ in a monotonic way, whereas $f_{2}$ changes from $1.79 \mathrm{MHz}$ to $1.45 \mathrm{MHz}$.

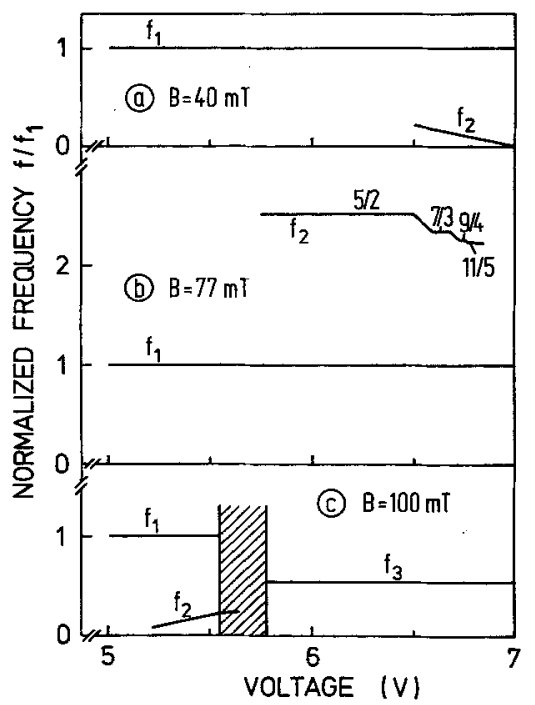

Fig. 2a-c. Frequencies of the current fluctuations in units of $f_{1}$ over the bias voltage range 5 to $7 \mathrm{~V}$ and for various magnetic fields $B$ : a $B=40 \mathrm{mT}$ : quasiperiodic behavior; b $B=77 \mathrm{mT}$ : frequency-locking states; c $B=100 \mathrm{mT}$ : Ruelle-TakensNewhouse scenario; the dashed area denotes the voltage range of chaos
At $B=100 \mathrm{~m} T$ the fluctuations again change their character drastically. A Ruelle-Takens-Newhouse scenario is found in agreement with previous observations [12].

Up to $5.21 \mathrm{~V}$, only the fundamental oscillation is found with frequency $f_{1}=1.07 \mathrm{MHz}$. The fractal dimension of the oscillation $d$, determined by the method of Grassberger and Procaccia [30] with embedding dimensions up to 14 , is about $d=1.0$. At higher voltages, a second oscillation arises again, whose frequency $f_{2}$ is now smaller than $f_{1}$ increasing continuously from $86 \mathrm{kHz}$ at $5.21 \mathrm{~V}$ to $225 \mathrm{kHz}$ at $5.55 \mathrm{~V}$. The Poincaré sections show closed curves due to a torus type attractor and the fractal dimension is about $d=2$ in this voltage interval. At $5.55 \mathrm{~V}$ chaos sets in as indicated by the hatched region in Fig. $2 \mathrm{c}$. In the course of the transition into the chaotic regime, the broad-band noise increases by $65 \mathrm{~dB}$ dominating the power spectra. The fractal dimension is $d=2.7$ at onset of chaos and rises slightly with increasing voltage. Above $5.8 \mathrm{~V}$ a new frequency $f_{3}$ determines the current fluctuation which remains constant at $f_{3}=547 \mathrm{kHz}$ up to $7 \mathrm{~V}$. The oscillation again has a fractal dimension $d=1$ showing that no other fundamental mode is present.

In summary, four different types of current oscillations have been observed: (i) regular relaxation oscillation at one fundamental frequency; (ii) quasiperiodic behavior with two independent frequencies; (iii) frequency-locking of two commensurate frequencies at several low integer ratios; (iv) Ruelle-TakensNewhouse scenario.

In Fig. 3 the observed types of post-breakdown current oscillation are visualized in a magnetic-fieldvoltage plane. Regular oscillations occur in a wide area of that plane shown by the shaded area. Quasiperiodic behavior could be observed in a narrow interval of voltage and low magnetic field $(B=16 \mathrm{mT})$ or in a

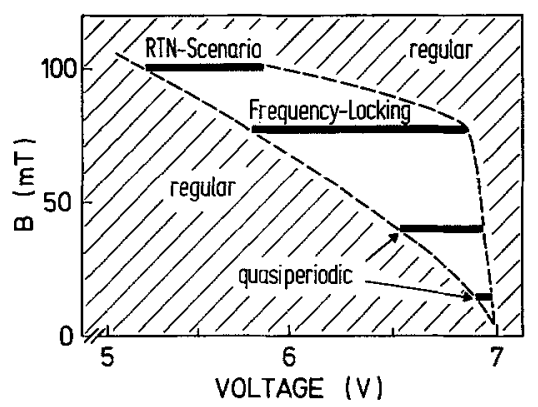

Fig. 3. The different types of current oscillations located in the magnetic-field-voltage plane. The sequence of quasiperiodic behavior, frequency-locking states and Ruelle-TakensNewhouse (RTN) scenario occurs in a window surrounded by regular oscillations 
wider range of the bias voltage at $B=40 \mathrm{mT}$ (see Figs. $2 \mathrm{a}$ and 3). At intermediate magnetic fields frequencylocking was obtained in a wide voltage range and for higher magnetic fields a Ruelle-Takens-Newhouse scenario governs the transition to chaos in a different interval of voltage. The dashed lines in Fig. 3 may serve as a guide to the eye for separating areas of regular oscillations at only one fundamental frequency from those where two or more additional frequencies or even chaos appear.

Regular oscillations result from the existence of instabilities, whereas quasiperiodic behavior and frequency-locking may be understood in terms of the circle-map theory by a second oscillatory process with increased coupling strength to the first one. The Ruelle-Takens-Newhouse scenario is likely if a third oscillation process arises and forces the quasiperiodic oscillations into chaos.

The magnetic field strength controls the type of oscillation via a second oscillatory process and its coupling strength to the fundamental process.

\section{Discussion}

The most obvious process that can lead to a regular oscillation in a wide range of voltage and magnetic field $B$ is a longitudinal fluctuation in the current density. As shown by Schöll [22] such fluctuations may be caused by dielectric relaxation and multilevel impact ionization of donors. The underlying idea is the following: Trapping of the injected current carriers increases the internal electric field, thus impact ionization of shallow impurities is enhanced and more free carriers are generated. Dielectric relaxation then lowers the internal electric field, therefore impact ionization becomes weak and recombination more probable. With trapping this process is periodically repeated.

Applying a magnetic field to the sample has many effects on its transport properties. Freeze-out of carriers in shallow donors and the increased magnetoresistance of the material may lower the impact ionization rate and therefore lower the dielectric relaxation time, reflected by a reduced frequency for the regular oscillation in agreement with our experimental results. The Lorentzian force also deflects the carrier trajectories thus lowering the mean free path.

Besides that, a second oscillatory process may be caused by energy relaxation in the energy distribution of the carriers. The underlying idea for that process is this: An applied electric field in a semiconductor may lead to an asymmetric Boltzmann distribution in the energy of the free carriers [31]. Electrons in the Boltzmann tail with a high kinetic energy can generate more free electrons by impact ionization of shallow donors than low energy electrons. Thus the Boltzmann tail is reduced and the concentration of low energy electrons is increased by both the inelastic scattering of high energy electrons to lower energies and by the impact ionization generation of additional free carriers [26-28]. These additional electrons reduce the internal electric field and hence lower the voltage at the sample. So by the increased carrier concentration the electronelectron interaction and the scattering at ionized impurities is increased, producing a reduction of the mean free path of carriers. The gain in kinetic energy of accelerated low energy electrons is reduced by the energy dependent scattering rate and the lower voltage across the sample. However, the high energy electrons may be accelerated until they loose energy either to the phonon system $[23,24]$, or, more likely, by impact ionization of shallow donors. This autocatalytic process results in a high current density with a thin Boltzmann tail and low impact ionization probability. Almost all shallow donors have been ionized. Therefore relaxation and recombination to the unoccupied donors becomes dominant. Now the free carrier concentration will be reduced and the voltage increased. Electron-electron interactions and scattering at impurities are less likely and the mean free path becomes longer again. The electrons may gain kinetic energy through acceleration caused by the high electric field. The distribution in the high energy tail becomes stronger and thus, with impact ionization, the process starts again. This carrier-concentration-dependent process is controlled by the magnetic field via the mean free path since the deflection of electron trajectories keeps the electrons longer in spatial regions of high scattering rates at ionized donors.

Quasiperiodic behavior may be understood by the absence of coupling, or only a weak coupling between two oscillatory processes. The observed quasiperiodic current fluctuations stem from the dielectric relaxation process and the energy relaxation process as discussed above. In terms of the circle-map theory, mode-locking states can be identified as Arnold tongues arising with an increased coupling strength [32]. The two oscillatory processes are coupled by a nonlinear impact ionization mechanism and in addition its frequencies and amplitudes are controlled by the magnetic field. For stronger magnetic fields, the shift of the energy levels of shallow donors can no longer be neglected. This will result in different generation-recombination kinetics. Thus quasiperiodic behavior may be driven to chaos by a third oscillatory process induced by the nonlinear generation-recombination kinetics. This sequence of successively appearing incommensurate frequencies and chaos with variation of the bias voltage can be described as a Ruelle-TakensNewhouse scenario, obtained in the present $\mathrm{n}-\mathrm{GaAs}$ sample at $B=100 \mathrm{mT}$. 


\section{Conclusion}

In conclusion, we have analyzed for the first time the sequence of spontaneous current fluctuations in $\mathrm{n}$-GaAs controlled by a weak external magnetic field $B$. We find regular oscillation, quasiperiodic behavior of two characteristic oscillations, frequency-locking of these two oscillations and chaos with a Ruelle-TakensNewhouse scenario. This sequence was discussed in terms of circle-map theory with two physical oscillatory mechanisms, due to dielectric relaxation and energy relaxation in the distribution of the carriers leading to an asymmetric Boltzmann distribution which depends on the free carrier concentration and on the electric field. The coupling between the two oscillatory processes is controlled and adjusted by the magnetic field and voltage bias.

Acknowledgements. We thank E. Schöll for helpful discussions and E. Bauser, Max-Planck-Institut für Festkörperforschung, Stuttgart, for providing the samples. Financial support by the Deutsche Forschungsgemeinschaft is gratefully acknowledged.

\section{References}

1. S.H. Koenig: Phys. Rev. 110, 986 (1958)

2. P.I. Oliver: Phys. Rev. 127, 1045 (1962)

3. R. Obermaier, W. Böhm, W. Prettl, P. Dirnhofer: Phys. Lett. A 105, 149 (1984)

M. Weispfenning, I. Hoeser, W. Böhm, W. Prettl, E. Schöll: Phys. Rev. Lett. 55, 754 (1985)

4. E. Schöll: Z. Phys. B 46, 23 (1982); 48, 153 (1982); 52, 321 (1983)

5. G.N. Maracas, W. Porod, D.A. Johnson, D.K. Ferry, H. Goronkin: Physica 134 B, 276 (1985)

6. S.B. Bumelione, Yu.K. Pozhela, K.A. Pyragas, A.V. Tamasevicius: Physica 134 B, 293 (1985)

7. S.W. Teitsworth, R.M. Westervelt, E.E. Haller: Phys. Rev. Lett. 51, 825 (1983)

S.W. Teitsworth, R.M. Westervelt: Physica 23 D, 181 (1986)

8. G.A. Held, C. Jeffries, E.E. Haller: Phys. Rev. Lett. 52, 1037 (1984)

G.A. Held, C. Jeffries: Phys. Rev. Lett. 55, 887 (1985)

G.A. Held, C.D. Jeffries: Proceedings of the International Symposium of Synergetics at Schloss Elmau, Bavaria, 6-11 May 1985

9. D.G. Seiler, C.L. Littler, R.J. Justice, P.W. Milonni: Phys. Lett. A 108, 462 (1985)

10. J. Peinke, A. Mühlbach, Z.P. Huebener, J. Parisi: Phys. Lett. A 108, 407 (1985)

J. Peinke, B. Röhricht, A. Mühlbach, J. Parisi, Ch. Nöldeke, R.P. Huebener, O.E. Rössler: Z. Naturforsch. 40a, 562 (1985) J. Peinke, A. Mühlbach, B. Röhricht, B. Wessely, J. Mannhart, J. Parisi, R.P. Huebener: Physica 23 D, 176 (1986)
B. Röhricht, B. Wessely, J. Peinke, A. Mühlbach, J. Parisi, R.P. Huebener: Physica 134 B, 281 (1985)

J. Peinke, J. Parisi, A. Mühlbach, R.P. Huebener: Z. Naturforsch. 42a, 441 (1987)

J. Peinke, J. Parisi, B. Röhricht, B. Wessely, K.M. Mayer: Z. Naturforsch. 42a, 841 (1987)

U. Rau, J. Peinke, J. Parisi, R.P. Huebener, E. Schöll: Phys. Lett. A 124, 335 (1987)

11. B. Röhricht, B. Wessely, J. Parisi, J. Peinke: Appl. Phys. Lett. 48, 233 (1986)

12. A. Brandl, T. Geisel, W. Prettl: Europhys. Lett. 3, 401 (1987)

13. E.G. Gwinn, R.M. Westervelt: Phys. Rev. Lett. 57, 1060 (1986); 59, 157 (1988)

14. K. Aoki, T. Kobayashi, K. Yamamoto: J. Phys. Soc. Jpn. 51, 2373 (1982)

K. Aoki, K. Yamamoto: Phys. Lett. A 98, 72 (1983)

K. Aoki, K. Miyamae, T. Kobayashi, K. Yamamoto: Physica 117, $118 \mathrm{~B}, 570$ (1983)

K. Aoki, O. Ikezawa, N. Mugibayashi, K. Yamamoto: Physica 134 B, 288 (1985)

15. S.W. Teitsworth, R.M. Westervelt: Phys. Rev. Lett. 56, 516 (1986)

16. R.M. Westervelt, S.W. Teitsworth: Physica 23 D, 187 (1986)

17. G.A. Held, C. Jeffries: Phys. Rev. Lett. 56, 1183 (1986)

18. K. Aoki, N. Mugibayashi, K. Yamamoto: Phys. Scr. T 14, 76 (1986)

19. K. Aoki, O. Ikezawa, K. Yamamoto: J. Phys. Soc. Jpn. 53, 5 (1983); Phys. Lett. A 98, 217 (1983); 106, 343 (1984)

20. S.W. Teitsworth, R.M. Westervelt: Phys. Rev. Lett. 53, 2587 (1984)

S.W. Teitsworth, B.A. Lynn, R.M. Westervelt: Phys. Scr. T 14, 71 (1986)

21. R.M. Westervelt, S.W. Teitsworth: J. Appl. Phys. 57, 5457 (1985)

R.M. Westervelt, S.W. Teitsworth, E.G. Gwinn: Phys. Scr. T 14, 65 (1986)

22. E. Schöll: Proceedings of the IV International Conference on Hot Electrons in Semiconductors, Physica 134 B, 271 (1985) E. Schöll: In Festkörperprobleme 26, edn. ed. by P. Grosse (Vieweg, Braunschweig 1986) pp. 309-333

E. Schöll: Phys. Rev. B 34, 1395 (1986)

E. Schöll: Nonequilibrium Phase Transitions in Semiconductors. 1st edn. (Springer, Berlin, Heidelberg 1987) pp. 223-285

23. B. Röhricht, J. Parisi, J. Peinke, O.E. Rössler: Z. Phys. B 65, 259 (1986)

24. E. Schö1l, J. Parisi, B. Röhricht, J. Peinke, R.P. Huebener: Phys. Lett. A 119, 419 (1987)

25. S.M. Abdurakhmanov, V.V. Vladimirov, V.N. Gorshkov: Solid St. Commun. 56, 399 (1985)

26. E. Schöll, W. Quade: J. Phys. C: Solid State Phys. 20, L 861-L 867 (1987)

27. F. Schlögl, E. Schöll: To be published

28. E. Schöll: Physica D and J. Phys. Chem. Solids (to be published)

29. P. Cvitanovic, B. Söderberg: Phys. Scr. 32, 263 (1985)

30. P. Grassberger, I. Procaccia: Phys. Rev. Lett. 50, 346 (1983)

31. C.J. Stanton, J.W. Wilkins: Phys. Rev. B 35, 9722 (1987)

32. M.H. Jensen, P. Bak, T. Bohr: Phys. Rev. A 30, 1960 (1984) 